\title{
International E-Learning Awards, Academic Division - 2013 Winners
}

The International E-Learning Awards, Academic Division recognize the best uses of technology for educational purposes in three subcategories: e-learning, mobile learning, and blended learning. All submissions are evaluated by the IELA Awards Committee, who look for a variety of attributes. These include, among others, educational soundness and effectiveness, usability, and overall significance.

The 2013 award winners were announced at our Awards Ceremony in Kazan, Russia at the ICL Conference on September 26, 2013. Information regarding the submission process and deadlines for the 2014 Awards, for both the Academic and Business categories, may be found under the Awards Program tab at www.ielassoc.org.

\section{We are pleased to recognize the 2013 Academic Division award winners:}

Winner, E-Learning: "iLearn-Pedes," Children's Hospital of E. Ontario and University of Ottawa, Canada. Representative: Toby Audecent.

Winner, Blended Learning: "Design of Mobile Learning - a blended course for K-12 teachers," KTH Royal Institute of Technology, Sweden. Representative: Stefan Stenbom.

Runner-Up, E-Learning: "ANTH202: Introduction to culture," eConcordia, Canada. Representative: Patrick Devey.

Runner-Up, Blended Learning: "LEED Project Experience," Everblue, United States. Representative: Amy Molloy.

Honorable Mention, E-Learning: "Learn and Cooperation Platform of the BMBF funding priority, "Center for Learning and Knowledge Management (ZLW), RWTH Aachen University and Chair of Information Systems I - Innovation \& Value Creation, Friedrich-Alexander-University Erlangen-Nuremberg, Germany. Representative: Tobias Vaegs.

Honorable Mention, E-Learning: "Language Teaching and Language Learning in a 3D Immersive Virtual Environment, " Algonquin College Language Institute, Canada. Representative: Dmitri Priven.

Honorable Mention, E-Learning: "KMUT - Platform for Project Management," Berlin School of Economics and Law, Germany. Representative: Julia Gunnoltz.

Honorable Mention, E-Learning: "Standardised Numeracy Assessment Process (SNAP)," University of Chester (SNAP Services), United Kingdom. Representative: Kieran Kelly.

Honorable Mention, Blended Learning: "EDIT 202," University of Alberta, Canada. Representatives: Representatives: Carol Tonhauser, Anwen Burk, Sherri Fricker, Kim Peacock, and Janet Welch.

Honorable Mention, Blended Learning: "CompEduHPT," KTH, Sweden. Representative: Torsten Fransson.

Honorable Mention, Mobile Learning: "Qatar University Mobile Learning Project," Qatar University, Qatar. Representative: Mohammed Samaka.

International E-Learning Association

c/o Kaleidoscope Learning, 304 Park Avenue South, $11^{\text {th }}$ Floor, New York, NY 10010,

Phone: +1 646 397-3710 Fax: +1 212 679-2738

info@ielassoc.org www.ielassoc.org 